\title{
The nucleon recoil effect in antikaon-deuteron scattering at threshold
}

\author{
V. Baru*, \\ Forschungszentrum Jülich, Institut für Kernphysik (Theorie) and Jülich Center for Hadron \\ Physics, D-52425 Jülich, Germany and \\ Institute for Theoretical and Experimental Physics, \\ B. Cheremushkinskaya 25, 117218 Moscow, Russia \\ E-mail: v.baru@fz-juelich.de \\ E. Epelbaum \\ Forschungszentrum Jülich, Institut für Kernphysik (Theorie) and Jülich Center for Hadron \\ Physics, D-52425 Jülich, Germany and \\ Helmholtz-Institut für Strahlen- und Kernphysik (Theorie), \\ Bethe Center for Theoretical Physics, Universität Bonn, D-53115 Bonn, Germany \\ E-mail: e.epelbaumefz-juelich.de
}

\section{A. Rusetsky}

Helmholtz-Institut für Strahlen- und Kernphysik (Theorie),

Bethe Center for Theoretical Physics, Universität Bonn, D-53115 Bonn, Germany

E-mail: rusetsky@itkp.uni-bonn.de

\begin{abstract}
We investigate the role of the nucleon recoil for antikaon-deuteron scattering using EFT. In particular, we show that the leading correction to the static term that appears at order $\xi^{1 / 2}$ (where $\xi=m_{K} / M_{N}$ ) cancels completely in the double scattering process. We calculate the higher order recoil corrections and study the pattern of convergence of the expansion method. We find that the recoil correction to the double scattering process is of order of $10-15 \%$ of the static contribution.
\end{abstract}

International Workshop on Effective Field Theories: from the pion to the upsilon

February 2-6 2009

Valencia, Spain

\footnotetext{
${ }^{*}$ Speaker.
} 


\section{Introduction}

A combined analysis of pionic hydrogen and pionic deuterium, which has been carried out recently, see e.g. Ref [1], has marked a significant progress towards a precise extraction of the pion-nucleon interaction parameters at threshold. One may expect that, in analogy to the above case, the properties of the $\bar{K} N$ interaction could be investigated by using a combined analysis of kaonic hydrogen and kaonic deuterium. The data on this sort of bound systems are provided by the ongoing experiment of DEAR/SIDDHARTA collaboration, which plans to measure the 1s energy level shift and width of kaonic hydrogen and kaonic deuterium eventually with an accuracy of several $\mathrm{eV}$ [2]. Note however that in contradistinction to the $\pi N$ case the $\bar{K} N$ scattering length is known to be large (of order of $1 \mathrm{fm}$ ) and strongly absorptive. Thus, in the kaonic case we have to solve a system of four Deser-type equations to determine the real and imaginary parts of the $\bar{K}^{-} p$ $\left(a_{p}\right)$ and $\bar{K}^{-} d\left(A_{K d}\right)$ scattering lengths independently:

$$
\begin{aligned}
& \Delta E_{1 s}-i \frac{\Gamma_{1 s}}{2}=-2 \alpha^{3} \mu^{2} a_{p}\left(1-2 \mu \alpha(\ln \alpha-1) a_{p}\right) \\
& \Delta E_{1 s}^{d}-i \frac{\Gamma_{1 s}^{d}}{2}=-2 \alpha^{3} \mu_{d}^{2} A_{K d}\left(1-2 \mu_{d} \alpha(\ln \alpha-1) A_{K d}\right),
\end{aligned}
$$

where $\mu\left(\mu_{d}\right)$ stands for the reduced mass of the $\bar{K} N(\bar{K} d)$ system. In the next step, one has to express the quantities $a_{p}$ and $A_{\bar{K} d}$ through the isoscalar and isovector S-wave $\bar{K} N$ scattering lengths $b_{0}, b_{1}$, which are defined in the isospin-symmetric world. The $K^{-} p$ scattering length, $a_{p}$, up to isospin symmetry breaking effects (ISB) that are derived in Ref. [3] and found to be significant, is just a linear combination of $b_{0}$ and $b_{1}: a_{p}^{s y m}=b_{0}-b_{1}{ }^{1}$. The situation is totally different for the kaonic deuterium, even in the absence of isospin breaking. During the last few decades, the problem of $\bar{K} d$ scattering at low energy has been studied thoroughly within the framework of Faddeev equations. However, the results of these calculations are of no direct use in the analysis of the SIDDHARTA data. In particular, these calculations do not provide an explicit relation of $A_{\bar{K} d}$ and $b_{0}, b_{1}$, which is needed for the analysis. On the contrary, the multiple scattering series for the $\bar{K} d$ scattering length (see, e.g., [5, 6]) is well suitable for the analysis. It has been pointed out in Ref. [6] that the non-relativistic effective field theory (EFT) provides an ideal tool to produce the multiple-scattering expansion. The reason why the multiple-scattering expansion for the $\bar{K} d$ scattering is useful, is due to the presence of two distinct momentum scales. Whereas the $N N$ interactions and 3-body $\bar{K} N N$ interactions are mediated at large distances by the one-pion exchange, the dominant long-distance contribution to the $\bar{K} N$ scattering comes from the two-pion exchange. For this reason, one may treat $\bar{K} N$ interactions as point-like, whereas $N N$ and $\bar{K} N N$ interactions will be described by non-local potentials. It is well known that the $\bar{K} N$ scattering lengths are large due to the presence of the subthreshold $\Lambda(1405)$ resonance, and the multiple-scattering series does not converge. Therefore the full infinite set of Feynman diagrams needs to be summed up to get a correct result for the $\bar{K} d$ amplitude. It was shown in Ref. [6] that the EFT calculation designed in this manner at leading order reproduces the results of the multiple scattering theory derived in Ref. [5]. Note however that to arrive at this result the nucleon kinetic energies have been neglected in

\footnotetext{
${ }^{1}$ Note that an extraction of the $K^{-} p$ scattering length derived within chiral unitary approaches from fits to available low-energy $K^{-} p$ scattering data has been done in Ref. [4].
} 


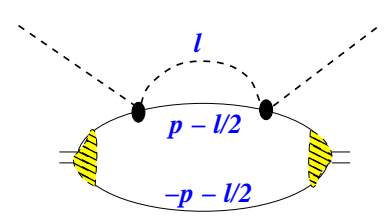

a)

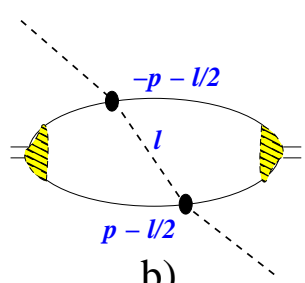

b)

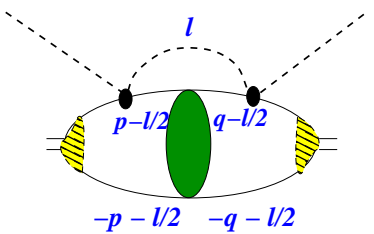

c)

Figure 1: Diagrams of double meson scattering off nucleons in the deuteron

the propagators of the Feynman diagrams, i.e. the so-called fixed center approximation (FCA) was employed. The validity of this approximation is the central question that needs to be addressed to enable a reliable determination of the $\bar{K} N$ scattering lengths from the data. Indeed, due to a large mass of the kaon, the parameter $m_{K} / M_{N}$ that controls the effect of the nucleon recoil is also large $(\sim 0.5)$. Thus, it is natural to conceive that the inclusion of the nucleon recoil would affect the results of the combined analysis of kaonic atoms. On the other hand, it is known that the FCA results of the multiple scattering theory are in a surprisingly good agreement with the calculations based on the Faddeev equations (see e.g. Ref. [7] for a detailed discussion of this topic) which indicates the appearance of some sort of cancellation between different recoil contributions. In this work we investigate the role of the nucleon recoil for $\bar{K} d$ scattering within EFT. The key assumption for the study is that the expansion in $\xi=m_{K} / M_{N}$ is perturbative for each particular diagram, even if the multiple scattering series are not. We will verify this assumption by explicit calculations of the recoil effect in the double scattering process.

\section{What do we know about nucleon recoil?}

Historically, the appearance of sizable cancellations in the case of the $\pi d$ scattering length was first pointed out in the papers by Kolybasov et al. [8] and, independently, by Fäldt [9] where it was claimed that the naive static term provides a good approximation for rescattering effects. Recently, the role of the recoil effects was investigated for $\pi d$ scattering and for the reaction $\gamma d \rightarrow \pi^{+} n n$ within EFT [10, 11]. It was shown, in particular, that the 3-body singularity that occurs in the $\pi N N$ intermediate state is the origin of non-analytic corrections to the static term of order of $\sqrt{m_{\pi} / M_{N}}$. It was also found that there is a direct connection between the role of the recoil effect and the Pauli selection rules for the intermediate NN state. In the case when the S-wave NN interaction is forbidden by the Pauli principle, the leading $\sqrt{m_{\pi} / M_{N}}$ correction cancels in the diagrams of double $\pi N$ scattering (diagrams a) and b) in Fig.1, thus resulting in the small net contribution. The situation is different in case when the S-wave $\mathrm{NN}$ interaction is allowed (see diagram c) in Fig. 1). First, the leading $\sqrt{m_{\pi} / M_{N}}$ correction does not vanish here (the contributions from diagrams a) and b) appear with the same sign). That is why it was concluded in $[10,11]$ that the recoil effect should be significant in this case. Secondly, the additional diagram with the S-wave NN interaction in the intermediate state must be also taken into account in the calculation. In the process $\gamma d \rightarrow \pi^{+} n n$, the intermediate NN interaction appears to be in the ${ }^{1} S_{0}$ partial wave. Since NN interaction in the ${ }^{1} S_{0}$ partial wave differs considerably to the one in the ${ }^{3} S_{1}$ partial wave, there is no a priori reason to expect any kind of cancellation between the recoil correction from diagrams similar to a) and b) and the contribution of diagram c). However, for $\pi d$ and $K d$ scattering the intermediate 
and final state NN interaction occurs in the same channel $\left({ }^{3} S_{1}-{ }^{3} D_{1}\right)^{2}$. Therefore, a combined consideration of all diagrams of Fig.1 is needed to conclude about the recoil effect. Note that the Pauli-allowed recoil correction for $\pi d$ scattering goes along with the isoscalar $\pi N$ scattering length squared which makes this effect negligible. This is, however, not the case for $K d$ scattering where both the isoscalar and isovector interactions are of a similar (and large) size.

\section{3. $\bar{K} d$ scattering: Recoil effect in the double scattering process}

Despite the well-known fact that the multiple-scattering series for the $\bar{K} d$ scattering are nonperturbative, at the first stage of our investigation we concentrate on the double-scattering diagram. The aim is to demonstrate the technique used to obtain a systematic expansion of any Feynman diagram in (generally non-integer) powers of the parameter $\xi$. To this end, we apply the perturbative uniform expansion method presented in Ref. [12]. The same results can be also obtained using the threshold expansion method of the Feynman diagrams in EFT developed in Ref.[13]. First, it can be shown that our amplitude for the double (see Fig.1) as well as multiple scattering process is expanded in powers of $\sqrt{\xi}$

$$
M=M_{s t}+\sqrt{\xi} M_{1}+\xi M_{2}+\xi^{3 / 2} M_{3}+\cdots
$$

where $M$ is related to the $\bar{K} d$-scattering length via $M=16 \pi M_{N}(1+\xi / 2) A_{\bar{K} d}$. The validity of this expansion and the pattern of convergence for the double scattering will became clear later in this paragraph. To calculate the amplitudes $M_{i}$ we follow the procedure of Refs.[12, 13]. In particular, for double scattering one gets (see Ref.[10] for more details)

$$
\begin{gathered}
M=M_{a}+M_{b}+M_{c}=2 M_{N}(8 \pi(1+\xi))^{2}\left\{b_{0}^{2}\left(I_{s t}+I_{0}+I_{N N}+\Delta I_{s t}\right)-3 b_{1}^{2}\left(I_{s t}-I_{1}+\Delta I_{s t}\right)\right\}, \\
I_{0(1)}=\int \frac{d^{3} p d^{3} l}{(2 \pi)^{6}}\left[\Psi^{2}\left(\vec{p}+\frac{\vec{l}}{2}\right) \pm \Psi\left(\vec{p}+\frac{\vec{l}}{2}\right) \Psi\left(\vec{p}-\frac{\vec{l}}{2}\right)\right]\left[\frac{1}{l^{2}+\xi\left(2\left(p^{2}+\gamma^{2}\right)+l^{2} / 2\right)}-\frac{1}{l^{2}(1+\xi)}\right], \\
I_{N N}=\frac{\xi}{M_{N}} \int \frac{d^{3} p d^{3} q d^{3} l}{(2 \pi)^{9}} \frac{\Psi\left(\vec{p}+\frac{\vec{l}}{2}\right) \Psi\left(\vec{q}+\frac{\vec{l}}{2}\right) M_{N N}(p, q, E(l))}{\left[l^{2}+\xi\left(2\left(p^{2}+\gamma^{2}\right)+l^{2} / 2\right)\right]\left[l^{2}+\xi\left(2\left(q^{2}+\gamma^{2}\right)+l^{2} / 2\right)\right]}, \\
I_{s t}=\int \frac{d^{3} p d^{3} l}{(2 \pi)^{6}} \frac{\Psi\left(\vec{p}+\frac{\vec{l}}{2}\right) \Psi\left(\vec{p}-\frac{\vec{l}}{2}\right)}{l^{2}} ; \quad \Delta I_{s t}=-\frac{\xi}{1+\xi} I_{s t}
\end{gathered}
$$

where $\Psi$ is the deuteron wave function, $M_{N N}$ is the invariant NN amplitude ${ }^{3}, \gamma^{2}=M_{N} \varepsilon_{d}$ with $\varepsilon_{d}$ being deuteron binding energy, and $E(l)=\varepsilon_{d}+\frac{l^{2}}{2 m_{K}}+\frac{l^{2}}{4 M_{N}}$. Here, $I_{s t}$ corresponds to the LO static (FCA) result when all $\xi$ corrections are dropped. The recoil corrections corresponding to the Pauli-allowed (forbidden) S-wave NN intermediate state in diagrams a) and b) of Fig.1 are represented by the integrals $I_{0}$ and $\Delta I_{s t}\left(I_{1}\right.$ and $\left.\Delta I_{s t}\right)$. For the Pauli-allowed NN state there is also a contribution of the diagram c) given by the integral $I_{N N}$. Thus, let us define the recoil corrections as $\Delta I_{1}=-I_{1}+\Delta I_{s t}$ and $\Delta I_{0}=I_{0}+I_{N N}+\Delta I_{s t}$ for isovector and isoscalar $\bar{K} N$ interactions, respectively. There are three relevant regimes in these integrals:

1) the regime of a 3-body singularity (small $l$ )

\footnotetext{
${ }^{2}$ Note that the relevant momenta in the integrals are of order of $m_{\pi}$. Therefore the contribution of the D-waves should be significantly suppressed as compared to the S-wave part.

${ }^{3}$ On shell $M_{N N}$ is related to the scattering amplitude $f=(k \cot (\delta)-i k)^{-1}$ via $M_{N N}(k, k, E(k))=16 \pi M_{N} f(k)$
} 

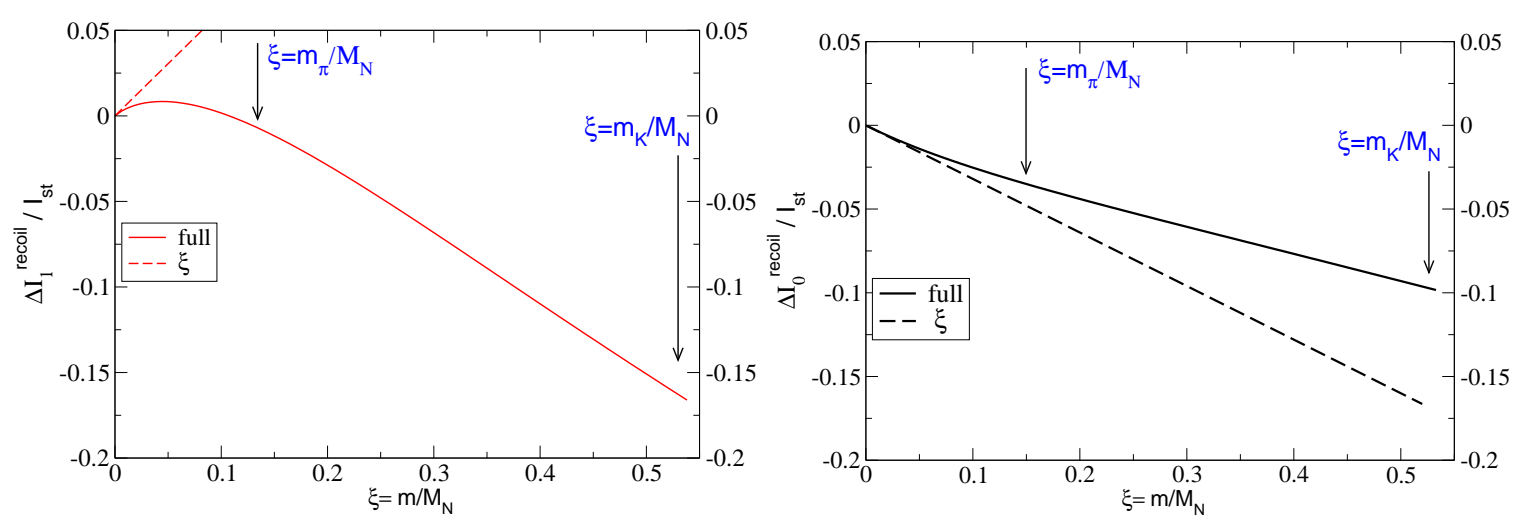

Figure 2: Recoil corrections in the double scattering process for the isovector (left panel) and isoscalar (right panel) cases. The notation of the curves is given in the text. The arrows indicate the results for $\pi d$ and $\bar{K} d$-scattering

$$
\frac{l^{2}}{2 m_{k}} \simeq \frac{p^{2}}{2 M_{N}} \Longrightarrow l \sim \sqrt{\xi} p, \quad p \sim\langle 1 / r\rangle_{w f}
$$

where \langle\rangle$_{w f}$ denotes averaging over the deuteron wave functions. In this regime, the integrals generate terms with non-integer powers of $\xi\left(\sqrt{\xi}, \xi^{3 / 2}, \ldots\right)$ in Eq.(3.1).

2) The heavy-baryon regime (large $l$ )

$$
l \sim p \sim\langle 1 / r\rangle_{w f}
$$

In this regime, the integrals produce terms with integer powers of $\xi$.

3) The intermediate regime, in which $\sqrt{\xi} p \ll l \ll p$.

In the current calculation we employ NN interaction in the separable form which allows for an analytic study of the recoil corrections. In this case the deuteron wave function has a Hulthen form and its D-wave component is neglected

$$
\Psi(p)=N g(p)\left(p^{2}+\gamma^{2}\right)^{-1}, g(p)=\left(p^{2}+\beta^{2}\right)^{-1}, \text { with } N=\sqrt{8 \pi \gamma \beta(\gamma+\beta)^{3}} .
$$

Performing the expansion of the integrals in each regime it can be easily demonstrated that there is a complete cancellation of the recoil corrections at order $\sqrt{\xi}$. One gets at this order

$$
\begin{aligned}
I_{0(1)} & =-2 \xi \int \frac{d^{3} p}{(2 \pi)^{3}}\left[\Psi^{2}(\vec{p}) \pm \Psi^{2}(\vec{p})\right]\left(p^{2}+\gamma^{2}\right) \int \frac{d^{3} l}{(2 \pi)^{3}} \frac{1}{l^{2}} \frac{1}{\left.l^{2}+2 \xi\left(p^{2}+\gamma^{2}\right)\right)} \\
& = \begin{cases}-\frac{\sqrt{\xi}}{\sqrt{2} \pi} \int \frac{d^{3} p}{(2 \pi)^{3}} \Psi^{2}(\vec{p}) \sqrt{p^{2}+\gamma^{2}} & \text { for isoscalar case } \\
0 & \text { for isovector case. }\end{cases}
\end{aligned}
$$

The integral $I_{N N}$ at the same order gives

$$
I_{N N}=\frac{\sqrt{\xi}}{\sqrt{2} \pi} \int \frac{d^{3} p}{(2 \pi)^{3}} \Psi^{2}(\vec{p}) \sqrt{p^{2}+\gamma^{2}} .
$$

Thus, one gets $I_{1}=0$ and $I_{0}+I_{N N}=0$, i.e. there is no recoil corrections at order $\sqrt{\xi}$. Note that for $\pi d$-scattering a similar result was obtained in Ref. [9] using a potential approach. Thus, the recoil 


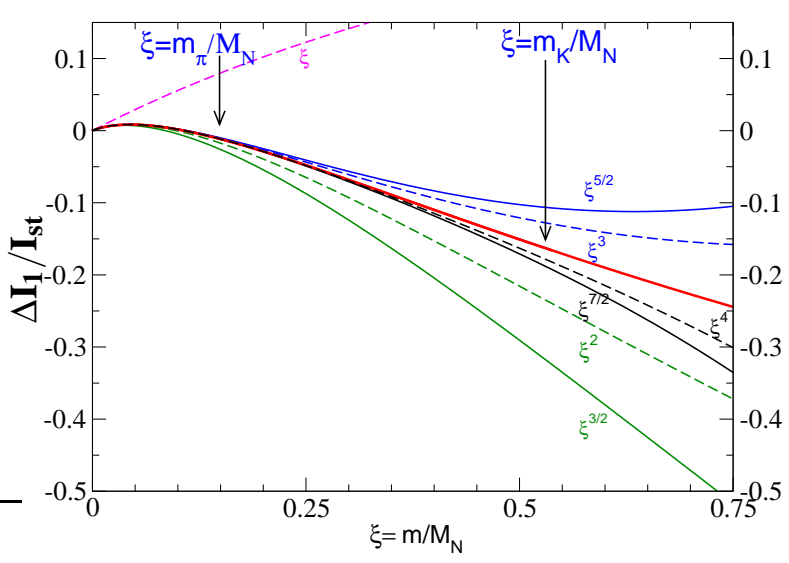

Figure 3: Recoil corrections in the double scattering process for the isovector $\bar{K} N$ interaction. Convergence of the expansion in $\xi$. The notation of curves is explained in the text. The arrows indicate the results for $\pi d$ - and $\bar{K} d$-scattering.

corrections to the static term both for isoscalar and isovector $\bar{K} N$ interaction will start from the term linear in $\xi$. In Fig. 2, we give the results for the recoil correction (in units of the static term $I_{\mathrm{st}}$ ) for isovector (left panel) and isoscalar (right panel) contributions as a function of $\xi$. The results of our full numerical calculation without expanding in $\xi$ are shown by the solid curves. Surprisingly even for $\bar{K} d$ scattering the nucleon recoil effect turns out to be not that large as one could a priori expect. As can be seen from the figure the nucleon recoil for the double scattering process amounts just to $10-15 \%$ of the static contribution. To understand the smallness of the effect more explicitly let us compare the results of the full calculation with those of our EFT calculation. Our results at order $\xi$ are shown by the dashed lines. Fig. 2 shows that for the isovector case the full recoil correction changes its sign in the interval considered, and that the linear approximation in this case fails to describe the total result. On the other hand, the recoil correction in the isoscalar case has a constant sign, and the linear approximation yields reasonable description. To see how does the expansion in $\xi$ converge in the case of the isovector $\bar{K} N$ interaction, we have calculated higher-order terms in $\xi$. The results are given in Fig. 3. The thick red line is again the full result, solid lines represent the results calculated up to and including some non-integer power of $\xi$, whereas the results shown by dashed lines include, in addition, the next integer power of $\xi$. Fig. 3 demonstrates that already at order $\xi^{2}$ one gets the bulk of the effect whereas the order $\xi^{4}$ provides a very good approximation to the underlying result for $\bar{K} d$ scattering. It is seen that there is a huge cancellation between the results at leading integer $(\xi)$ and leading non-integer $\left(\xi^{3 / 2}\right)$ orders that even leads to a change of sign for the recoil effect. Further, while improving convergence at smaller $\xi$, an inclusion of new non-integer terms results in the oscillatory behavior around the full result at larger $\xi$. Thus, we conclude that the smallness of the net recoil effect is accounted for by specific cancellations amongst different recoil corrections.

\section{Summary}

We have studied the nucleon recoil effect for $\bar{K} d$ scattering using EFT. Specifically, using the expansion method of the Feynman diagrams in EFT, we have calculated recoil corrections to the double scattering process in a systematic expansion in the half-integer powers of the parameter $\xi=M_{K} / m_{N}$. It is shown that the leading correction to the static term, which emerges at order $\xi^{1 / 2}$, cancels completely both for isoscalar and isovector types of $\bar{K} N$ interaction. The origin of the cancellation for the isovector case can be explained by the Pauli principle whereas for the isoscalar 
case it stems from the orthogonality of the bound state (deuteron) and continuum ( $N N$ intermediate state) wave functions in the ${ }^{3} S_{1}$ partial wave. The coefficients of higher order terms in the expansion appear to be of a natural size and the series converges even for the value of $\xi$ corresponding to the physical kaon mass. The relative smallness of the net recoil effect can be explained by specific cancellations of recoil corrections at different orders. A more detailed discussion of this and other aspects can be found in the upcoming publication [14].

\section{Acknowledgments}

We would like to thank U.-G. Meißner and A. Gal for interesting discussions. The work of E.E. and V.B. was supported in parts by funds provided from the Helmholtz Association to the young investigator group "Few-Nucleon Systems in Chiral Effective Field Theory" (grant VH-NG-222). This research is part of the EU Integrated Infrastructure Initiative Hadron Physics Project under contract number RII3-CT-2004-506078. Work supported in part by DFG (SFB/TR 16, "Subnuclear Structure of Matter"), by the DFG-RFBR grant (436 RUS 113/991/0-1) and by the Helmholtz Association through funds provided to the virtual institute "Spin and strong QCD" (VH-VI-231). V. B. acknowledges the support of the Federal Agency of Atomic Research of the Russian Federation. A.R. acknowledges financial support of the Georgia National Science Foundation (Grant \#GNSF/ST08/4-401).

\section{References}

[1] S. R. Beane et ., Nucl. Phys. A 720, 399 (2003) [hep-ph/0206219]; U.-G. Meißner et al., Phys. Lett. B 639, 478 (2006) [nucl-th/0512035]; V. Lensky et al., Phys. Lett. B 648, 46 (2007), [nucl-th/0608042]; V. Baru et al., Phys. Lett. B 659, 184 (2008) [arXiv:0706.4023 [nucl-th]]; V. Baru et al., In the Proceedings of MENU 2007, Julich, Germany, Sep 2007, 127 [arXiv:0711.2743 [nucl-th]]; J. Gasser et al., Phys. Rept. 456, 167 (2008), [arXiv:0711.3522 [hep-ph]].

[2] G. Beer et al. [DEAR Collaboration], Phys. Rev. Lett. 94, 212302 (2005); C. Curceanu, talk given at EXA 2008, Vienna, Austria, Sep 2008, http://www.oeaw.ac.at/smi/event/exa08

[3] U.-G. Meißner, U. Raha and A. Rusetsky, Eur. Phys. J. C 35349 (2004) [hep-ph/0402261]; R. H. Dalitz and S. F. Tuan, Ann. Phys. 8 100, (1959).

[4] B. Borasoy, U. -G. Meissner and R. Nissler, PRC 74, 055201 (2006) [hep-ph/0606108].

[5] S. S. Kamalov, E. Oset and A. Ramos, Nucl. Phys. A 690, 494 (2001) [nucl-th/0010054]; R. Chand and R. H. Dalitz, Annals Phys. 20 (1962) 1.

[6] U.-G. Meißner, U. Raha and A. Rusetsky, Eur. Phys. J. C 47, 473 (2006) [nucl-th/0603029]; A. Rusetsky, Proceedings of MENU 2007, Julich, Germany, Sep 2007, 162

[7] A. Gal, Int. J. Mod. Phys. A 22, 226 (2007) [nucl-th/0607067].

[8] V. M. Kolybasov and V. G. Ksenzov, Zh. Eksp. Teor. Fiz. 7113 (1976)

[9] G. Fäldt, Phys. Scripta 16, 81 (1977)

[10] V. Baru et al., Phys. Lett. B 589, 118 (2004) [nucl-th/0402027].

[11] V. Lensky et al., Eur. Phys. J. A 26, 107 (2005) [nucl-th/0505039].

[12] R. F. Mohr et al., Annals Phys. 321, 225 (2006) [nucl-th/0509076]; R. F. J. Mohr, PhD thesis, [nucl-th/0306086]

[13] M. Beneke and V. A. Smirnov, Nucl. Phys. B 522, 321 (1998) [hep-ph/9711391].

[14] V. Baru, E. Epelbaum and A. Rusetsky, in preparation 